\title{
El orden de palabras en los fueros de Alcaraz y de Alarcón
} Mónica Castillo Lluch

\section{Citer ce document / Cite this document :}

Castillo Lluch Mónica. El orden de palabras en los fueros de Alcaraz y de Alarcón. In: Cahiers de linguistique hispanique médiévale. $\mathrm{N}^{\circ} 21,1996$. De la variation linguistique et textuelle. En I'honneur de Jean Roudil. pp. 273-291;

doi : https://doi.org/10.3406/cehm.1996.878

https://www.persee.fr/doc/cehm_0396-9045_1996_num_21_1_878

Fichier pdf généré le 01/10/2019 


\section{EL ORDEN DE PALABRAS EN LOS FUEROS DE ALGARAZ Y DE ALARGÓN}

El género jurídico es, dentro del conjunto de la prosa medieval castellana, uno de los más relevantes y, sin embargo, el carácter lingüístico de este tipo de escritura y el alcance de su aportación dentro del marco de la formación de la lengua están todavía por evaluar ${ }^{1}$. En buena medida esta carencia se debe a que en ocasiones el investigador ni siquiera dispone del material editado, lo que entorpece todo intento de estudio en relación con los textos. Este era el caso de los fueros que integran la familia de Cuenca-Teruel. El profesor Roudil, consciente de que se imponía como tarea de primera necesidad la edición de textos para aclarar todo tipo de incógnitas relacionadas con la tradición filológica, la lengua y la historia de esta familia de fueros, en 1968 edita los de Alcaraz y de Alarcón. Con este trabajo arduo y generoso, Jean Roudil pone al alcance del investigador jurista o lingüista un material estudiado y editado impecablemente, abriendo así una vía de investigación en

1) La lingüística histórica se ha ocupado muy parcialmente del estudio de los textos jurídicos antiguos. Lapesa ha dedicado varios trabajos $(1948,1963,1972$ y 1973) al análisis de la lengua de los fueros de Avilés, Madrid, Valfermoso de las Monjas y Villavaruz de Rioseco, centrándose en su descripción fonética y en algunos aspectos morfológicos y léxicos, pero concediéndole una atención limitada a su sintaxis. El comportamiento evasivo por parte de los lingüistas con respecto al análisis de la sintaxis foral resulta muy común. Así, Gutiérrez Cuadrado (1974: 10) en su estudio del Fuero de Béjar pretende «estudiar con brevedad y claridad la fonética y la morfología del texto, dentro de las perspectivas tradicionales. [...] El estudio sintáctico lo hemos dejado aparte de momento, porque estamos convencidos de que sólo se puede abordar provechosamente con instrumentos modernos. " $\mathrm{La}$ reacción extrema, también corriente, consiste en descartar definitivamente trabajar con documentos forales. Tal es el caso, por ejemplo, de Hinojo Andrés (1988 : 439), quien en su estudio general sobre el orden de palabras en el castellano medieval toma corno corpus todos los textos en prosa dc la Crestomatía pidaliana « excepto los Fueros por el carácter peculiar de su lengua ». 
sendos campos de estudio. Volviendo al problema de partida, el propio Roudil, en su introducción, subraya la importancia de definir la naturaleza de la lengua jurídica del siglo XIII y sus aportaciones a la creación de la prosa castellana.

Las páginas que siguen pretenden ser una pequeña contribución al campo de estudio lingüístico abierto por Jean Roudil con esta edición. Nos centraremos aquí en su interés lingüístico, analizando, en concreto, la influencia sintáctica del original latino en los dos fueros.

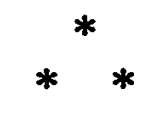

Los textos jurídicos medievales -contratos de compraventa, donaciones, testamentos, fueros, etc.- anteriores a 1250 presentan tal interacción del latín y el romance, que lingüísticamente las dos lenguas coexistentes merecen ser estudiadas independientemente. Los fueros de Alcaraz y de Alarcón ${ }^{2}$ fueron redactados a finales del s. XIII, momento en el que se afianzaba la práctica del castellano como lengua de cultura, ya superada la reforma cluniacense y sobre todo gracias a la labor de Alfonso X, razón por la cual no presentan ya, en apariencia, ningún elemento latino (no hay léxico aislado en latín, ni fórmulas introductorias en esa lengua...); es decir, externamente son textos enteramente romances. No obstante, al tratarse de adaptaciones del texto latino del fuero de Cuenca ${ }^{3}$, hay que tener presente que es probable que escondan influencias del texto original.

2) Después de la reconquista de Alarcón (1184) y de Alcaraz (1213) realizada por Alfonso VIII, estas localidades, desprovistas de legislación, adoptaron el fuero de Cuenca, nueva capital del Reino. El fuero de Cuenca fue redactado bajo Alfonso VIII y este mismo fue quien lo otorgó a Alcaraz y Alarcón en la primera mitad del siglo XIII. La redacción romance de los textos data de finales del s. XIII. El fuero de Alcaraz fue redactado en 1296 por Bartolomé de Uzeda. La escritura del códice utilizado para la edición es de finales del s. XIII. El fuero de Alarcón se conserva en un solo manuscrito también con escritura de finales del s. XIII.

3) Tomaremos como fuente de referencia el texto latino del Forum Conche, aunque no está probado que sea este realmente el modelo de nuestros dos fueros. Por el contrario, parece que el fuero de Alcaraz sea traducción del Forum Consocre. El fuero de Alarcón es más dificil de emparentar: por su plan no puede ser una traducción del Forum Consocre; posee similitudes con el fuero Alcázar, pero por razones textuales no puede ser hijo de este ni viceversa. Por todo este origen tan oscuro de los dos fueros nos permitiremos la simplificación de adjudicarle al Fonum Conche la paternidad de ambos. La misma simplificación adopta Jean Roudil (1968:10) y la justifica del siguiente modo: 
Desafortunadamente, aún hoy siguen sin descifrar numerosos aspectos de la tradición textual de la familia de fueros de CuencaTeruel $^{4}$. Centrándonos en los fueros de Alcaraz y de Alarcón, aún se desconoce si estos son adaptación directa de un original latino de la tradición del Forum Conche $e^{5}$, si los adaptadores utilizaron para su elaboración junto a la versión latina una romance o si copiaron directamente de una versión ya completamente romance. Estos elementos serían extremadamente útiles como base para un estudio lingüístico y sin duda son, desde el punto de vista filológico, los que exigen una investigación más urgente. Hasta qué punto son lingüísticamente independientes los adaptadores de nuestros fueros al traducir, es lo que vamos a intentar esclarecer en el presente artículo $y$, en función de las conclusiones que arroje este análisis, ofreceremos una hipótesis sobre la relación de cercanía o lejanía de los dos fueros con respecto a un posible original latino.

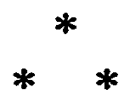

El hecho de encontrar en nuestros fueros hipérbatos a todas luces calcados del latín que en romance originarían frases agramaticales, nos proporciona ya el primer indicio de que sin duda hay en las adaptaciones al castellano una notable influencia de la sintaxis del original latino ${ }^{6}$. P. ej. :

Prendre comme point de départ ce texte [el Forum Conche] ne signifie nullement qu'il a été la source de toutes les traductions, mais c'est une base de référence, choisie parce que:

a) Elle est la seule, avec le Forum Turolij, à être conservée intégralement encore de nos jours ;

b) Elle a fait l'objet d'une transcription sérieuse - celle de Ureña y Smenjaud -, alors que les malheureuses éditions du Forum Turolï sont inutilisables et ne correspondent nullement aux exigences philologiques modernes ;

c) Elle doit permettre, par une étude comparative très poussée de retracer la généalogie de la famille de Cuenca-Teruel et de voir quels groupes de fueros s'en écartent, supposant ainsi d'autres voies de transmission.

Para este punto cfr. además García Gallo (1956: 438-439, 445) quien señala que los investigadores han supuesto tradicionalmente que el Fuero de Cuenca fue el modelo que adaptaron o copiaron los fueros extensos de la misma región, objetando que se impone reconsiderar la tradición textual de esta familia de fueros.

4) Ureña y Smenjaud (1935: GVI) en la introducción a su trabajo proporciona una descripción de esta familia de fueros. Véase también García Gallo (1956: 430-440) y Roudil (1968: 10).

5) Fn adelante, Forum Conche $=C$, Fuero de Alcaraz $=A z$ y Fuero de Alarcón $=A n$.

6) Hemos utilizado la edición de Ureña y Smenjaud. Nuestras citas corresponden a la Forma Sistemática del Forum Conche. 
$A z:$ De aquel que casa crebantare agena $(147,7)$

$C:$ De eo qui domum uiolauerit alienam (1265)

Podemos considerar esta sintaxis como consecuencia de un desliz de atención por parte del adaptador de $A z$ en la traducción ${ }^{7}$. Si bien es verdad que este tipo de construcción dentro de las dos obras es relativamente excepcional, el ejemplo anterior no deja de constituir una prueba explícita de que el orden que presenta es fruto de una influencia sintáctica del original latino del que es traducido. Sin embargo, aparte de estos indicios, para llegar a conclusiones fundadas acerca del verdadero alcance de esta influencia sintáctica latina, se impone un análisis profundo del tejido básico de estos escritos.

7) En esta ocasión, la lección de An es perfectamente gramatical :

$A n:$ E tod aquel que casa agena quebrantare $(147,19)$

Pocas líneas después, $A z$ retoma la sintaxis propia del romance sin el hipérbaton latino: $A z:$ Otrossi, qual quier que casa agena encendiere $(149,1)$

No obstante, por regla general, en los dos fueros no se suele quebrantar la sintaxis del castellano :

$C:$ De eo qui maurum percusserit alienum (2711-2712)

$A z:$ De aquel que moro ageno firiere o matare $(281,1)$

$A n:$ Del que moro ageno firiere $(218,19)$

Dos lecciones más con hipérbatos agramaticales aparecen en el Fuero de Alarcón, pero esta vez su origen es misterioso si lo comparamos con las originales del Forum Conche, que no presentan esta alteración de orden :

(1) An: Et si por uentura el alcayat o omne del alcayat danno fiziere alguno o calonna, $(92,20)$

$C: \mathrm{Si}$ alcayat, aut homo ipsius aliquem dampnum aut calumpniam fecerit, (164166)

(2) An: E si alguno armas saccare uedadas, $(214,19)$

$C$ : si quis arma pohibita extraxerit (2627-8)

Aquí, la incógnita de la tradición nos impide postular que estas lecciones sean particulares de $A n$, puesto que queda la posibilidad de que el texto latino del que copie An realmente contuviera estas dos estructuras con hipérbaton que no presenta en este caso $C$.

La versión de $A z$ de (1) es correcta : Mas si el alcayd o el sennor o sus omnes algun danzo o alguna calonna fizieren, $(92,10)$. Sin embargo, cabe la posibilidad de que la estructura de An hubiera sido motivada por una confusión del caso sintáctico de aliquem. Esta palabra, en vez de ser considerada como lo que es, un adjetivo en acusativo, calificativo de dampnum, se interpreta como un dativo, aliqui, originando una versión con un nuevo constituyente, un objeto indirecto : si el alcayat danno fiziere a alguno. La equivocación de la función sintáctica quedaría justificada por la corrupción de la declinación latina en ese momento y la falta de garantía sintáctica que ofrecían, por tanto, las desinencias de la época. Por otra parte, la interpretación de la lección alguno como un objeto indirecto en $A n$ se podria explicar por la asimilación de la $a$ de la preposición de objeto indirecto a la $a$ de alguno. 
Por el carácter propio de las normas jurídicas, que contienen un juicio hipotético por el que se vincula un supuesto de hecho a una consecuencia jurídica $(p \rightarrow q)$, abundan en nuestros fueros ejemplos sobre todo de dos tipos de oraciones: las subordinadas circunstanciales condicionales y las subordinadas adjetivas especificativas.

Ya en una primera lectura de los fueros, aparte de los órdenes agramaticales citados arriba, llama la atención la elevada proporción de oraciones de verbo finial, sobre todo entre las subordinadas de las que se acaba de hablar. En esta ocasión nos centraremos en el análisis sintáctico de las condicionales, y en particular en la posición relativa que en ellas presentan los elementos oracionales básicos: sujeto (S), objeto (O) y verbo (V).

\section{ANÁLISIS DE LAS SUBORDINADAS CONDIGIONALES}

\section{Orden básico en la prosa medieval española}

Nos interesa aquí exponer someramente la ordenación fraseal predominante de la prosa antigua para más tarde comparar la sintaxis de los fueros de Alcaraz y de Alarcón con la practicada en un texto contemporáneo escrito originalmente en castellano. En particular, nos centraremos en el orden de las oraciones subordinadas, dado que nuestro objeto de análisis en los fueros serán las circunstanciales condicionales.

La diferencia de organización básica entre las oraciones principales o independientes y las subordinadas viene siendo motivo de estudio desde el siglo pasado, cuando los problemas de orden de palabras en general comenzaron a acaparar la atención de los gramáticos. La investigación al respecto ha confirmado que desde el latín se aprecia mayor proporción de verbos finales en subordinada que en principal ${ }^{8}$. Si el indoeuropeo era una lengua

\footnotetext{
8) Cfr. p. ej, Adams (1977: 69) : «Yet in Latin of all periods, including that of late vulgar
} 
$\mathrm{OV}^{9}$ y el latín arcaico seguía ofreciendo el mismo orden, ya en el latín de Plauto, de la época preclásica, se observa masivamente la distribución VO, sobre todo en las oraciones independientes, en tanto que en las subordinadas se continúa practicando un orden de verbo final ${ }^{10}$. Los resultados estadísticos del célebre estudio de Linde $(1923)^{11}$ demostraron definitivamente que, tanto en latín clásico - en el que por convención literaria seguía utilizándose el orden OV - como en el vulgar - donde se impuso plenamente la ordenación VO -, la secuencia OV era más frecuente en subordinada que en principal. Franzén (1939: 136-138), por su parte, llegó a la misma constatación ${ }^{12}$.

Dentro del ámbito de las lenguas románicas se ha confirmado que existe igualmente mayor tendencia hacia una colocación del verbo en posición final en periodo hipotáctico que en paratáctico ${ }^{13}$. Los estudios en particular sobre el orden de palabras en español medieval proporcionan resultados que junto a nuestros datos personales utilizaremos en adelante para exponer la disposición básica de la prosa antigua. En esta ocasión recurriremos fundamentalmente a trabajos que aportan frecuencias estadísticas de los distintos órdenes en subordinada y en principal ${ }^{14}$, puesto que

texts, the verb showed a considerably greater tendency to gravitate to the final position in subordinate clauses than it did in main clauses » y Ramsden (1963:51): « in all periods of Latin, final verb position was more usual in subordinate clauses than in main clauses ».

9) Véase Lehmann (1974).

10) Véase Adams (1976).

11) Dichos resultados sobre las frecuencias de oraciones con verbo final son: César: O. Prpal $84 \% /$ O. Subord. $93 \%$ e Itinerarium Egeriae (S. IV-V): O. Prpal $25 \%$; O. Subord. $37 \%$.

12) Hay que añadir a este respecto, no obstante, que otros autores no ven tan clara una diferencia real entre oraciones principales y subordinadas, argumentando que se trataría exclusivamente de una pequeña diferencia de grado en las estadísticas. Así, por ejemplo en opinión de Pinkster (1990: 168) :

For subordinate clauses this is less clear. For Caesar it has been demostrated that in subordinate clauses finite verbs are placed at the end even more often that in main sentences [...] but this is merely a difference of degree. In other authors, too, only a small difference has been found, which need not necessarily be related to the difference main sentence : subordinate clause.

13) Marchello-Nizia (1995) proporciona una lista bibliográfica puesta al día y muy completa de los estudios sobre el orden de palabras en francés. Entre los autores que constatan mayor proporción de verbo final en subordinada figuran Thurneysen (1892), Foulet (1924 : 58) y Marchello-Nizia (1955 : 78).

14) Lamentablemente, existen estudios que analizan el orden de palabras en español 
necesitamos manejar cifras concretas para poder establecer posteriormente una comparación entre los resultados generales de los escritos en prosa de la época y los que arrojan los textos de los Fueros de Alcaraz y de Alarcón. A este respecto hay que lamentar que el trabajo comparativo de Crabb (1955) - que tan valioso nos hubiera sido aquí por los datos estadísticos que ofrece - se ciña exclusivamente al estudio de las oraciones independientes y deje de lado un análisis de las subordinadas. Entre los lingüistas que proponen cifras concretas de la diferente ordenación en los dos tipos de oraciones, figuran John England (1980), quien ha examinado la posición del objeto directo medieval y Javier Elvira (1987) que se ha ocupado de la colocación del verbo en español antiguo. Los resultados convergentes de ambos autores nos serán de gran valor en este estudio; fusionados y sintetizados son los siguientes :

1. La posición VO estaba ya arraigada en español antiguo en todo tipo de oración (England, $1980: 4$ y Elvira, $1987: 73$ ).

2. La posición del sujeto con respecto al verbo era, por el contrario, más libre (England, 1980: 4) ${ }^{15}$. En las oraciones principales se advierte una tendencia del verbo a situarse en posición inicial absoluta, lo que provoca la inversión del sujeto (VS) en más del $60 \%$ de los casos estudiados (Elvira, $1987: 75-77)^{16}$.

antiguo cuyos resultados estadísticos no podemos utilizar aquí, como por ejemplo el de Hinojo Andrés (1988) y el de Meyer-Hermann (1988), ya que estos autores no proporcionan datos desglosados de oraciones principales y subordinadas.

15) Company (1985-1986 : 78-79) defiende la misma idea :

Para la frase nominal objeto el orden no marcado es después del verbo, VO. [...] Un orden OV siempre se puede caracterizar como marcado en el español de los siglos XII al XV. [...] En el español de estos siglos, verbo y objeto presentan una unión muy estrecha - ampliando el término pudiera hablarse de cierta «cohesión » entre verbo y objeto -, mucho más estrecha que la existente entre sujeto y verbo y ambos constituyentes, verbo y objeto, parecen funcionar como un todo frente al sujeto.

16) Hay que considerar en este punto opiniones controvertidas como la de MeyerHermann (1988: 556, 559), quien en un estudio específico de la posición del sujeto en español antiguo, defiende que desde su formación el español estaba ya configurado como una lengua SVO. Precisamente basándose en los datos obtenidos de un examen de la posición relativa $S-V$ en los fueros de Salamanca, Zamora y Sepúlveda, sin observar diferencias entre oraciones principales y subordinadas, es como este autor llega a esta conclusión que aplica a toda la lengua medieval. Habría que reconsiderar el carácter general de esta deducción a la vista de la diferencia de ordenación entre la prosa narrativa, por ejemplo, descrita por Elvira como mayoritariamente representativa del orden VS en principales y la prosa jurídica, que, como vamos a ver, se decanta totalmente por la secuencia SV. Los factores sintácticos que provocan la inversión del sujeto son descritos por Elvira (1988). 
3. En las subordinadas se observan dos tendencias: 1) la proporción mayoritaria de VS de las principales disminuye o incluso se invierte (Elvira, 1987: 76-77) $;^{17}$ y 2) a menudo el verbo aparece en posición final absoluta precedido por su objeto (England, 1980 : 11 y Elvira, $1987: 77)$.

En adelante, describiremos la estructura básica de las subordinadas condicionales en textos prosísticos medievales con objeto de proporcionar una referencia comparativa con respecto a los fueros que nos ocupan. El artículo de England (1980: 11) nos ofrece resultados estadísticos específicos sobre el orden del objeto nominal en oraciones subordinadas condicionales de textos representativos del género del exemplum medieval ${ }^{18}$. Las frecuencias de OV en las oraciones condicionales son reproducidas a continuación :

\begin{tabular}{|l|c|c|c|c|c|c|c|}
\hline Type of clause & Calila & Engaños & Castigos & Lucanor & Gatos & $A B C$ & Espéculo \\
\hline Condition & $12,7 \%$ & $15,4 \%$ & $25,0 \%$ & $15,7 \%$ & $28,6 \%$ & $19,4 \%$ & $6,8 \%$ \\
\hline
\end{tabular}

Por nuestra parte, al analizar las subordinadas condicionales de un texto jurídico de la misma época de los fueros, como lo es la Segunda Partida, y de contratos de compraventa incluidos en los Documentos Lingǘsticos de España (D.L.E.) editados por Menéndez Pidal $^{19}$, de cronología más variada, hemos llegado a las siguientes conclusiones :

17) Herman (1954 : 239, 262, 283-284) en el análisis que realizó sobre el orden de palabras en los más antiguos textos en prosa franceses, confirma igulmente para el francés que mientras en las oraciones principales se da fluctuación entre los órdenes SV/VS, siendo a menudo VS el que se impone, en las oraciones subordinadas es mayoritaria la secuencia SV.

18) La muestra de England abarca el periodo de 1250-1450. Los textos que examina son El libro de Calila e Digna, El libro de los engaños, Castigos e documentos para bien vivir ordenados por el rey don Sancho IV, El Conde Lucanor, El libro de los gatos, Libro de los exemplos por a.b.c. y El espéculo de los legos.

19) Las ediciones manejadas son las de Juárez Blanquer y Rubio Flores (1991) y Menéndez Pidal (1966). Nuestros resultados son fruto de sondeos parciales a lo largo de las cien primeras páginas de la Segunda Partida y de toda la edición de los D.L.E. Del conjunto de condicionales halladas solo serán tomadas en cuenta las formadas como mínimo por dos de los constituyentes básicos de la oración -sujeto (S), verbo (V), objeto directo nominal (O)-. En la Segunda Partida de 148 condicionales trabajarcmos con 45 quc siguen el criterio anterior, y en los D.L.E. de 43 trabajaremos con 28. 


\section{Segunda Partida}

\begin{tabular}{|c|c|}
\hline SV & VS \\
\hline $89,3 \%$ & $10,7 \%$ \\
\hline
\end{tabular}

\begin{tabular}{|c|c|}
\hline VO & OV \\
\hline $81,8 \%$ & $18,2 \%$ \\
\hline
\end{tabular}

D.L.E.

\begin{tabular}{|c|c|}
\hline SV & VS \\
\hline $76,5 \%$ & $23,5 \%$ \\
\hline
\end{tabular}

\begin{tabular}{|c|c|}
\hline VO & OV \\
\hline $57,9 \%$ & $42,1 \%$ \\
\hline
\end{tabular}

\section{Orden de las subordinadas condicionales en los Fueros de Alcaraz y de Alarcón 20}

Sirvan como ilustración del conjunto de subordinadas circunstaciales condicionales de los dos fueros las siguientes oraciones :

$A z$ : Qve si por auentura, vezino de la cibdat omne estranno fallare en el termino de Alcaraz, $(83,11)$; Si alguno uiere a otro laurar en la heredat que el cuyda que es suya, $(106,2)$; Mas si uinna plantare, $(109,5)$; Et si alguno le tolliere el ganado, (121, 12); Si can o puerco la uinna dannare, $(135,7)$; Et si por auentura, despues del desposorio, el esposo al esposa o la esposa al esposo repoyare, $(174,9)$; Si el padre o la madre fijo trauiesso ouieren $(182,9)$; Et si alguna bestia a otra firiere o matare, $(209$, 2 ) ; Et si el moro de paz al cristiano firiere o matare, $(218,14)$; Si por auentura el omicida la fiadura de saluo negare, $(281,7)$.

$A n$ : si por auentura, vezino de la uilla fallare al estranno en el termino de Alarcon $(83,25)$; $\mathrm{Si}$ alguno uiere en su heredat a otro labrar, $(106,21)$; E si uinna plantare $(109,22)$; Et si alguno el ganadol tolliere, $(121,27)$; Si perro o puerco en uinna fallare, $(135,23)$; E si el esposo refusare al esposa, $(174,22)$; Si padre o madre ouieren fijo loco, $(182,24)$; Et si .I. ${ }^{\mathrm{a}}$ bestia firiere o matare otra bestia, $(209,12)$; E si el moro de paz matare o firiere christiano, $(218,32)$; Si por uentura el homizero la fiadura negare, $(281,23)$.

20) Hemos realizado un sondeo exhaustivo de las oraciones condicionales en las primeras doscientas páginas de la edición. La muestra está formada del siguiente modo : en $A z$ hay 602 condicionales, de las que solo se tomarán en cuenta 342, siguiendo los criterios formulados en la nota anterior para los ejemplos de la Segunda Partida y los D.L.E. An presenta 564 condicionales de las que seleccionamos 291. La diferencia cuantitativa entre lus dos textos se debe a que An traduce en ocasiones condicionales como adjetivas especificativas. 
Los resultados que arroja nuestro análisis de la distribución relativa $\mathrm{S}-\mathrm{V}$ y $\mathrm{V}-\mathrm{O}$ son los siguientes :

$A z$

\begin{tabular}{|c|c|}
\hline SV & VS \\
\hline $98,5 \%$ & $1,5 \%$ \\
\hline
\end{tabular}

\begin{tabular}{|c|c|}
\hline VO & OV \\
\hline $17,5 \%$ & $82,5 \%$ \\
\hline
\end{tabular}

$A n$

\begin{tabular}{|c|c|}
\hline SV & VS \\
\hline $92,7 \%$ & $7,3 \%$ \\
\hline
\end{tabular}

\begin{tabular}{|c|c|}
\hline VO & OV \\
\hline $39,8 \%$ & $60,2 \%$ \\
\hline
\end{tabular}

Dos observaciones se desprenden inmediatamente de estos resultados : 1) existe una diferencia con respecto al orden canónico que acabamos de exponer general al español antiguo y 2) se aprecia un comportamiento dispar de la posición relativa $\mathrm{O}-\mathrm{V}$ entre los dos fueros.

\section{a. Diferencia de la sintaxis de los fueros con respecto al orden básico del español medieval}

En cuanto al orden S - V, los dos fueros presentan un porcentaje casi totalitario del mismo - en ambos casos superior al $92 \%$ - de SV. Si bien veíamos más arriba que Elvira (1987: 77) advertía una tendencia mayor en las oraciones subordinadas en comparación con las principales en la práctica de un orden SV, este porcentaje nunca supera un $67 \%$ en el más radical de los $\operatorname{casos}^{21}$. En comparación con nuestros resultados, hay que señalar que las cifras de los fueros y de la Segunda Partida se asemejan bastante (SV : $A z: 98,5 \%, A n: 92,7 \%, P I I: 89,3 \%$ ), y que los D.L.E. (76,5\%), a pesar de presentar la secuencia SV en proporciones superiores a las

21) Los porcentajes de SV en oraciones subordinadas que proporciona Elvira (1987: 77) son : $27,6 \%$ cn La Fazienda de Ultramar, $29 \%$ en el Liber Regum, 58,3\% en la Primera Crónica General y $66,9 \%$ en la Historia Troyana. 
de la muestra de Elvira, no alcanzan los valores extremistas de $A z$ y de $A n$. Así pues, la casi absoluta organización SV - aunque no parece exclusiva de los fueros, ya que también presentan frecuencias elevadas de esta secuencia la Segunda Partida y los Documentos Lingǘsticos de España - puede contemplarse como un primer indicio singularizador de la sintaxis de $A z$ y $A n$.

Ahora bien, es en la comparación del orden relativo de $\mathrm{O}$ y $\mathrm{V}$ de nuestros fueros con el que presentan otros textos castellanos, donde las diferencias resultan más elocuentes. Hemos visto que el rasgo más afianzado de la sintaxis medieval es la ordenación VO y si tanto England (1980:4) como Elvira (1987: 77) señalan que la secuencia OV no es rara en subordinadas, la combinación inversa, VO, era, no obstante, la preponderante. Esto queda corroborado por las estadísticas proporcionadas por England (1980:11) para las subordinadas condicionales de los exempla (una media de $82,3 \%$ de VO) y las nuestras de la Segunda Partida (81,8\% de VO) y de los D.L.E. ( $57,9 \%$ de VO). Resulta, pues, extraordinario encontrarse ante unas estadísticas de distribución mayoritaria $\mathrm{OV}$ en subordinadas en escritos en prosa castellana del siglo XIII. Haciendo abstracción de la diferencia de frecuencias de $A z$ y $A n$, de la que nos ocuparemos más tarde, las cifras de OV en estos dos fueros representan una mayoría frente a VO. Es este el rasgo sustancial que particulariza la sintaxis de $A z$ y $A n$ en el marco de la lengua medieval.

A este respecto, hay que señalar que Lapesa (1948:106) en el apartado que dedica al análisis de la morfología y la sintaxis del fuero de Avilés, al tratar de la colocación del verbo en la oración, observa igualmente que «con notable frecuencia el verbo ocupa el final de la frase, o por lo menos se pospone al régimen y, si es auxiliar, al participio. " Tratándose de un texto foral, las condiciones de esta secuencia OV son similares a las que nosotros describimos: se da especialmente en oraciones encabezadas por un pronombre o adverbio relativo (« ome que mulier prenda... et arras li dedit», 63-64), en la mayoría de las hipótesis con si (« Si non per suo grado pausar, e a forcia pausar, deféndasi ", 7), en las construcciones de infinitivo con preposición (" per solar prender", 4) y en definitiva es más corriente en subordinada que en principal.

En resumen, la mayoría de SV y OV en los fueros de Alcaraz y de Alarcón es atípica en la práctica de la prosa medieval española, lo que nos conduce a la conclusión de que sintácticamente estos textos jurídicos reflejan una influencia muy marcada del original latino. 


\section{b. Diferencias entre Az y An}

La primera diferencia concierne a la posición relativa $\mathrm{S}-\mathrm{V}$ en $A z$ y $A n$. Si bien encontramos en $A n$ casi cinco veces más el orden VS que en $A z$, estas cifras no son muy significativas, dado el bajo número de ejemplos a partir del cual se han establecido los porcentajes : $1,5 \%$ en $A z=4$ ejs. y $7,3 \%$ en $A n=17$ ejs. Además, la mayor proporción de VS en $A n$ se explica fácilmente por la tendencia de este último a traducir participios pasivos latinos como sujetos activos en contra de la costumbre conservadora de $A z^{22}$. Es esta la razón principal por la cual se desequilibran las frecuencias entre los dos textos. Aparte, $A n$ improvisa en cuatro ocasiones un orden VS donde en latín era SV, que $A z$ respeta.

La verdadera desigualdad reside, sin embargo, en la posición de $\mathrm{V}$ y $\mathrm{O}$. En ambos textos es mayoritaria la organización OV, pero la frecuencia de $A z(82,5 \%)$ sobrepasa de un modo más que anecdótico la cifra de $A n(60,2 \%)$. Esto, en principio, evidenciaría que $A z$ sigue de modo más servil la sintaxis del original latino y que $A n$ se ha alejado más de ella. Con objeto de definir con cuánta frecuencia coinciden $A z$ y $A n$ con un supuesto original latino en cuanto al orden OV / VO hemos comparado nuestros fueros con el de Cuenca. A continuación se reproducen de forma tabular los resultados de la confrontación de los tres documentos :

\begin{tabular}{|c|c|c|}
\hline Solución sintáctica & en $A z$ & en $A n$ \\
\hline Conservación del orden OV lat. & $88 \% 23$ (143 casos) & $61 \%$ (76 casos) \\
Permutación OV lat. $\rightarrow$ VO rom. & $12 \%(19$ casos $)$ & $39 \%$ (48 casos) \\
Conservación del orden VO lat. & $100 \%(10$ casos $)$ & $80 \%$ (4 casos) \\
Permutación VO lat. $\rightarrow$ OV rom. & $0 \%(0$ casos $)$ & $20 \%$ (1 caso) \\
Greación orden OV $\varnothing$ lat. $24 \rightarrow$ OV) & 13 casos & 10 casos \\
Creación orden VO $(\varnothing$ lat. $\rightarrow$ VO) & 2 casos & 7 casos \\
\hline
\end{tabular}

22) P. ej.:

$C:$ Si tres fuerint diffidiati, (3609)

$A z:$ Si tres fueren desafiados $(261,2)$

$A n:$ Si .III. fueren los desafiados $(261,16)$.

23) Los porcentajes no observan los casos de traducción de OV/VO latinos por otra expresión sin objeto en romance.

24) «Ø lat. " representa los casos en los que en el original latino no existe un objeto directo, que es improvisado por el adaptador al traducir al romance. P. ej. :

$C:$ Quod nisi fecerit, (1695)

$A z:$ e si esto non fiziere, $(166,13)$ 
En resumen, podemos concluir a partir de estos datos estadísticos que :

$-A z$ sigue mayoritariamente el orden $\mathrm{OV}$ del original latino (143 veces $-88 \%$ ).

- Solo en pocas ocasiones (19 casos - $12 \%$ ) aventura un orden VO a partir de OV en latín.

- Cuando improvisa un objeto directo inexistente en latín, practica con más comodidad el orden OV (10 veces) que VO ( 2 veces).

- An sigue el original latino en el orden OV (76 ocurrencias $61 \%$ ) con menos frecuencia que $A z$.

- Con bastante asiduidad (48 veces - $39 \%$ ) traduce OV latino como VO.

- Improvisa indistintamente un objeto directo en orden VO / OV ( 7 y 10 casos respectivamente).

De todo esto se deduce que existe una diferencia en el grado de seguimiento del original latino por parte de los dos adaptadores : el de $A z$ es más servil calcando mecánicamente el orden latino de verbo final, lo que provoca una sintaxis extremista en cuanto a las secuencias OV; en $A n$ se aprecia una tensión : su adaptador, a la vez que mantiene muchos casos de OV latino, da muestras de autonomía dejándose llevar hacia un estilo más romance, traduciendo OV como VO en numerosas ocasiones.

\section{Argumentos adicionales que apoyan la influencia sintáctica latina.}

A los ya expuestos, podemos añadir otros argumentos que confirman la influencia sintáctica latina en nuestros fueros :

Lo dicho con respecto a las oraciones condicionales se hace extensible al resto de subordinadas. $\mathrm{El}$ orden $\mathrm{OV}$ se encuentra con las mismas frecuencias en las subordinadas adjetivas especificativas - que son las más numerosas junto con las condicionales -. P. ej. : el bibdo que fijos ouiere e muger tomar quisiere (An 187, 30).

Por otra parte, no son raros los casos de elisión del artículo allí donde el castellano normalmente lo presentaría, lo que ocasiona la aparición de nombres sin actualizar. En los ejemplos de las oraciones condicionales ofrecidos más arriba encontramos varios casos : 
$A z$ : Qve si por auentura, vezino de la cibdat omne estranno fallare en el termino de Alcaraz, $(83,11)$; Mas si uinna plantare, $(109,5)$; Si can o puerco la uinna dannare, $(135,7)$; Si el padre o la madre fijo trauiesso ouieren $(182,9)$;

$A n$ : si por auentura, vezino de la uilla fallare al estranno en el termino de Alarcon $(83,25)$; E si uinna plantare $(109,22)$; Si perro o puerco en uinna fallare, $(135,23)$; Si padre o madre ouieren fijo loco, $(182,24)$; E si el moro de paz matare o firiere christiano, $(218,32)$;

Refleja igualmente un influjo latino la traducción de sicut dictum est con el participio antepuesto al auxiliar (como dicho es). A finales del siglo XIII, fecha de composición de los fueros de Alcaraz y de Alarcón, el orden auxiliar - participio se había consolidado definitivamente, en particular cuando el tiempo compuesto figuraba precedido por otra palabra y no comenzaba frase ${ }^{25}$. La aparición del orden contrario debe ser analizada, pues, como ajena a la lengua romance de ese periodo. También en este caso $A n$ da pruebas de su autonomía con respecto al latín, traduciendo 9 veces como dicho es y 20 como es dicho ${ }^{26}$, mientras que $A z$ presenta 27 casos de participio antepuesto y solo 2 de orden contrario.

Finalmente, en las construcciones de verbo regente + infinitivo, las secuencias en las que el infinitivo precede al verbo conjugado aparecen con mayor frecuencia que en textos romances contemporáneos. De nuevo aquí estamos ante un calco sintáctico en la traducción de las expresiones correspondientes latinas, p. ej.: si probari potuerit $>$ si prouargelo pudieren 27 .

25) Es bien conocido el principio de la lengua antigua de que los auxiliares haber y ser cuando el tiempo compuesto en el que participaban comenzaba periodo, por su naturaleza átona, se posponían al participio. Ahora bien, ya desde Berceo se recogen más anteposiciones del auxiliar al participio que el orden contrario. Para este punto cfr., p. ej., Menéndez Pidal (1908: 412-413, § 205.9 y $1926: 399-340, \S 80.5)$, Lapesa (1942: 218, § 582) y González Ollé (1983:5).

26) Herman (1954: 242-245) también considera como una prueba de independencia del traductor antiguo la práctica del orden auxiliar - participio donde el latín utilizaba la secuencia contraria.

27) En relación con este punto, Lapesa (1948: 106) indica que en el fuero de Avilés se practican las dos sintaxis, sin señalar cuál es mayoritaria : "El infinitivo se antepone unas veces al verbo personal que lo rige, otras se pospone: «si lidiar non quisier», 66 ; "qui... sos peinos sacar li quisier», 56, frente a «fudiador quero dar», 20 ; «si pobre fidiador quesierit intrar ", 12. ". 


\section{CONGLUSIONES}

La comparación de la sintaxis de los fueros Alcaraz y de Alarcón 1) con la de otros textos romances de la época, 2) entre sí y 3) con el original latino del Forum Conche arroja las siguientes conclusiones :

- El porcentaje de subordinadas condicionales de verbo final en estos dos fueros es muy superior a la media de un texto romance.

$-A z$ practica una sintaxis más cercana a la del original latino, con una mayoría rotunda de condicionales de secuencia (S)OV. An en este sentido estaría más despegado del orden latino.

La sintaxis de los fueros de Alcaraz y de Alarcón está, pues, fuertemente impregnada de la influencia del original latino del que son adaptaciones. La frecuencia con la que presentan órdenes de SV y OV (en definitiva SOV) dista mucho de la que proporcionan otros escritos originales en romance de la época. Si bien Lapesa (1948: 107) rechaza la hipótesis de que la sintaxis de verbo final que presentan fueros como los de Avilés o Madrid pueda ser considerada como cultismo 28 , en nuestra opinión, no obstante, resulta lícito pensar que la conservación de una sintaxis latinizante estaba provocada en buena medida por la consideración de que ese estilo otorgaba prestigio a los textos, y que tal orden concedía, en definitiva, una garantía de texto culto. A este respecto nos interesa citar la impresión de Crabb, aplicada al descubrimiento por parte de Menéndez Pidal de una sintaxis latinizante en las glosas y hasta cierto punto en el Cantar de Mio Cid ${ }^{29}$. En palabras de Crabb (1955 : xii) :

The glosses were necessarily pedantic and Latin-conscious and one could scarcely hope to find reflected in them the true state

28) En palabras de Lapesa (1948: 107):

El apego a la construcción latina con el verbo al final no es consecuencia del cultismo, ya que se manifiesta en los textos vulgares más antiguos: domina en las Glosas Emilianenses y Silenses, en documentos del siglo XII, abunda en el Cantar de Mio Cid y se da en el Fuero de Madrid (anterior a 1202) en circunstancias idénticas y con igual profusión que en el Fuero de Avilés.

29) La observación de Menéndez Pidal (1926:397, § 80.1) es la siguiente :

El uso latino del verbo al final de la frase continúa muy arraigado en nuestro período [romance preliterario]. El atributo, el objeto directo o el complemento adverbial preceden ordinariamente al verbo: «dulce jet» GlEmil 117; « castigatore fueret » GISil 55, 292 ; 
of contemporary $\mathrm{O}[\mathrm{ld}] \mathrm{S}$ [panish] w[ord] o[rder]. To the contrary, the glossers might be expected to favor a w[ord] $o$ [rder] more typically Latin than Castilian. The end position of the verb which Menéndez Pidal cites is in fact the least favored $w$ [ord] $o[$ rder] type of all in the prose examined here.

A una conclusión similar llega England (1980), autor que examina la posición del objeto en exempla medievales y que comprueba en principio que el idioma en el que estaban escritos los textos originales a partir de los que se tradujeron los exempla no ejerce influencia alguna en la colocación del objeto ${ }^{30}$. Ahora bien, en contraste con esta generalidad en cuanto a la independencia sintáctica de los exempla con respecto a sus fuentes, constata England (1980 : 18-19) que el seguimiento del original latino en cuanto al orden OV en El espéculo de los legos se produce con mayor frecuencia en los pasajes traducidos de la Biblia o de auctores latinos que en el resto del texto, de carácter narrativo o didáctico :

This indicates a totally different approach on the part of the translator towards different sections of his material, according to content and source; the narratives and didactic comments of the compiler of the Latin Speculum Laicorum he makes conform to the Old Spanish V-O order, rather than attempt to reproduce the predominant $\mathrm{O}-\mathrm{V}$ order of the original [...]. The difference in approach [...] probably reflects the translator's respect for the language of the Bible and of the major auctores, in that he is prepared to alter the word order used by the original compiler, but reproduces that of sacred and authoritative texts.

Esta idea de que el género de los textos posee una incidencia en el seguimiento del original del que son traducciones, viene apoyada en nuestro caso por toda la carga de «sacralidad » que posee lo jurídico. El carácter prescriptivo de los fueros hace que el

30) Cfr. England (1980: 5-6) : « direct object position does not vary according to the source language of each text. " Este autor demuestra en particular que en El libro de los gatos y en El espéculo de los legos, traducciones de las Fabulae de Odo de Cheriton y del Speculum Laicorum respectivamente, realizadas en los siglos XIV y XV, existe una diferencia llamativa entre los originales latinos de orden mayoritario OV $(46,5 \%$ y $64,3 \%)$ y las correspondientes versiones romances, en las que el orden OV solo representa un 4,6\% $y$ un $4,1 \%$ en cada caso. England $(1980: 6)$ concluye que : "It thus seems likely that the translators of both texts recognised the differences in object position between Latin and Old Spanish, and deliberately eliminated the characteristic Latin O-V constructions in their translations. " 
respeto a las leyes y a todo el sistema jurídico se traslade a una veneración igual de solemne hacia su lenguaje. Por lo tanto, era poco esperable que los adaptadores de estos documentos se emanciparan de toda la tradición de los textos jurídicos en latín, para de repente organizar sus fueros de un modo independiente a los inmediatamente anteriores, escritos en el latín de los jurisconsultos de la decadencia.

Por otro lado, posiblemente el caso del adaptador de $A n$, en el que se percibe una clara tendencia a separarsc dcl orden de verbo final del latín, represente el principio del esfuerzo por el cual con el tiempo se superaría el compromiso del estilo latinizado. Otro tipo de razones, en relación con la tradición textual de los dos fueros, pueden formularse para explicar este comportamiento divergente del adaptador de $A n$ con respecto al de $A z$. ¿Utiliza el adaptador de $A z$ un texto latino como modelo original y el de $A n$ a la par una versión latina y otra romance, $o$ incluso solo una fuente romance? A juzgar por los datos lingüísticos parece ser así. El carácter probatorio de argumentos sintácticos para llegar a conclusiones válidas en la definición de la tradición textual puede estar sujeto a reservas, pero no puede negársele todo su valor.

Mónica Castillo Lluch

(Université Paris-XIII

S.E.M.H.)

\section{Referencias :}

ADAMS, James Noel (1976): «A typological approach to Latin wordorder », Indogermanische Forschungen Zeitschrift, LXXXI, pp. 70-99.

- (1977): The Vulgar Latin of the letters of Claudius Terentianus. Manchester, Manchester Universtity Press.

COMPANY, Concepción (1985-1986) : « Los futuros en el español medieval. Sus orígenes y su evolución ", Nueva Revista de Filología Hispánica, XXXIV, pp. 48-107.

CRABB, Daniel (1955): A comparative study of word order in old Spanish and old French prose works. Washington, The Catholic University of America Press. 
Elvira GonZÁlez, Javier (1987) : «Enclisis pronominal y posición del verbo en español antiguo » Epos, 3, pp. 63-79.

- (1988) : «La posición del sujeto en español antiguo ». Actas del I Congreso Internacional de Historia de la Lengua Española, I, Madrid, Arco Libros, pp. 339-349.

ENGLAND, John (1980): «The position of the direct object in old Spanish ». Joumal of Hispanic Philology, 5, pp. 1-23.

Foulet, Lucien (1924) : «L'accent tonique et l'ordre des mots. Formes faibles du pronom personnel après le verbe ». Romania, 50, pp. 54-93.

FRanZ.ÉN, Torsten (1939) : Etude sur la syntaxe des pronoms personnels sujets en ancien français. Uppsala.

García Gallo, Alfonso (1956) : «Aportación al estudio de los fueros » Anuario de Historia del Derecho Español, XXVI, pp. 387-446.

GONZÁlez OLLÉ, Fernando (1983) : «Enclisis pronominal en el participio de las perífrasis verbales ». Revista de Filología Española, LXIII, pp. 1-32.

Gutiérrez Guadrado, Juan (1974): Fuero de Béjar. Salamanca, Universidad de Salamanca.

Herman, József (1954) : «Recherches sur l'ordre des mots dans les plus anciens textes français en prose ». Acta Linguistica Academiae Scientiarum Hungaricae, IV, pp 69-94 y 351-382. (Reeditado en HERMAN, József (1990): Du latin aux langues romanes. Etudes de linguistique historique. Tübingen, Niemeyer, pp. 234-288.)

HINOJO ANDRÉS, Gregorio (1986): "El orden de palabras en la Peregrinatio Aetheriae ». Studia Zamorensia Philologica, VII, pp. 79-88. JUÁREZ BLANQUER, Aurora y Antonio RUBIO FLORES (eds.) (1991): Partida Segunda de Alfonso $X$ el Sabio. Manuscrito 12794 de la $B . \mathcal{N}$. Colección Romania, $\mathrm{n}^{\circ} 3$, Granada.

LehmanN, Winfred P. (1974) : Proto-Indoeuropean Syntax, Austin.

LAPESA, Rafael (1942): Historia de la lengua española. Madrid, Gredos. [Utilizo 9a ed. de 1986.]

- (1948): "Asturiano y provenzal en el Fuero de Avilés ». Acta Salmanticensia, II, $\mathbf{n}^{\circ}$ 4. Publicado en LAPESA, Rafael (1985): Estudios de historia linguḯstica española. Madrid, Paraninfo, pp. 53-122.

- (1963) : «El Fuero de Madrid » en El Fuero de Madrid. Madrid, ed. por el Ayuntamiento de Madrid, pp. 151-163. Publicado en LAPESA, Rafael (1985) : Estudios de historia lingüística española. Madrid, Paraninfo, pp. 157-166.

- (1972): «Los provenzalismos del Fuero de Valfermoso de las Monjas (1189) ». Hispanic Studies in honor of Edmund de Chasca, Philological Quarterly, 51, 1, pp. 54-59. Publicado en LAPESA, Rafael (1985): Estudios de historia linguïstica española. Madrid, Paraninfo, pp. 123-127.

- (1973) : «Rasgos franceses y occitanos en el lenguaje del Fuero de Villavaruz de Rioseco (1181)". Mélanges de linguistique française et de philologie et littérature médiévales offerts à monsieur Paul Imbs, Travaux de Linguistique et de Littérature, XI, 1, pp. 529-532. Publicado en LAPESA, Rafael (1985) : Estudios de historia lingüística española. Madrid, Paraninfo, pp. 128-131. 
LINDE, P. (1923) : «Die Stellung des Verbs in der lateinischen Prosa", Glotta, XII, pp. 151-178.

MARchello-NiziA, Christiane (1995) : L'évolution du Franģais : Ordre des mots, démonstratifs, accent tonique. Paris, Armand Colin.

MENÉndez PIDAL, Ramón (1908): Cantar de Mio Cid. Texto, gramática y vocabulario. Madrid, Espasa Calpe.

- (1926): Orígenes del español. Estado lingüístico de la Península Ibérica hasta el siglo XI. Madrid, Revista de Filología Española, Anejo I.

- (1966) : Documentos Lingǘsticos de España. Madrid, C.S.I.C.

MEYER-HERMANN, Reinhard (1988) : «La posición del sujeto en español antiguo y moderno (en comparación con el francés) ». Actas del I Congreso Internacional de Historia de la Lengua Española, I. Madrid, Arco Libros, pp. 541-562.

Pinkster, Harm (1990): Latin Syntax and Semantics. Londres, Routledge.

RAMSDEN, Herbert (1963) : Weak-pronoun position in the early romance languages, Manchester, Manchester University Press.

Roudil, Jean (1968) : Les fueros d'Alcaraz et d'Alarcón. Paris, Klincksieck.

Thurneysen, Rudolf (1892): "Zur Stellung des Verbums im Altfranzösischen ». Zeitschrift fiir Romanische Philologie, XVI, pp. 289307.

URENA y SMENJAUd, Rafael de (1935) : Fuero de Cuenca. (Formas primitiva y sistemática: texto latino, texto castellano y adaptación del Fuero de Iznatoraf). Madrid, Academia de la Historia. 